\title{
Energy Distribution of a Regular Class of Exact Black Hole Solutions
}

\author{
I-Ching Yang ${ }^{1}$, Chi-Long Lin $^{2}$, I. Radinschi ${ }^{3}$ \\ ${ }^{1}$ Department of Applied Science \\ and Systematic and Theoretical Science Research Group, \\ National Taitung University, Taitung, Taiwan 950, \\ icyang@nttu.edu.tw, \\ ${ }^{2}$ The National Museum of Natural Science, \\ Taichung, Taiwan 403, Republic of China, \\ and ${ }^{3}$ Department of Physics, "Gh. Asachi" Technical University, \\ Iasi, 700050, Romania \\ radinschi@yahoo.com
}

November 2, 2018

\begin{abstract}
In this paper we present the expressions for the energy of a regular class of exact black hole solutions of Einstein's equations coupled with a nonlinear electrodynamics source. We calculate the energy distribution using the Einstein, Weinberg and Møller prescriptions. We make a discussion of the results in function of two specific parameters, a sort of dipole and quadrupole moments of the nonlinear source $\alpha$ and $\beta$, and in addition a study of some particular cases is performed.
\end{abstract}

\section{INTRODUCTION}

Energy-momentum localization has presented a remarkable progress in the recent years, although a satisfactory generally accepted expression for energy distribution has not been found. As an illustration, we notice the pseudotensorial definitions [1]-[7] which have been used for computing the energy of $3+1$, $2+1$ and 2 dimensional geometries enlightening that different energy-momentum complexes can yield the same expression for energy distribution of a given spacetime [8]-[9]. These nontensorial quantities contain the contribution of the matter and non-gravitational fields and that of the gravitational field, and give rise of the problem of coordinate dependence. Among these energy-momentum prescriptions only the Møller's definition can be applied to any coordinate system [10]. Although the coordinate dependence, recently many interesting studies 
have been performed for exploring various geometries and making more accessible the issue of energy-momentum localization [8]-[10]. This involves understanding the pseudotensorial mechanism, how can be used for the evaluation of energy of different space-times and attempt to provide many illustrative examples. For the Einstein, Landau-Lifshitz, Papapetrou and Weinberg prescriptions the problem of coordinate dependence was partially solved in the case of the metrics of Kerr-Schild class [11]. Further, for a great number of space-times the energy-momentum complexes give the same results with their tele-parallel versions [12]. Chang, Nester and Chen in an interesting work [13] concluded that energy-momentum complexes are directly connected to quasilocal expressions for the energy-momentum, and each energy-momentum complex is associated with a legitimate Hamiltonian boundary term. The connection with the boundary conditions also implies that each expression for energy has a geometrically and physically significance. All these considerations point out the significance of the energy-momentum complexes and stress their usefulness for the localization of energy. Even the researchers are confident that the pseudotensorial mechanism gives meaningful result, the main question remains available, how the coordinate dependence could be overcome and a generally consistent formula for energy-momentum density developed. Moreover, for clarifying some weakness of the pseudotensorial definitions and point out their best properties future work is needed.

In this paper we evaluate the energy of a regular class of exact black hole solutions of Einstein's equations coupled with a nonlinear electrodynamics source [14] using the Einstein, Weinberg and Møller prescriptions. We show that the expression for the Weinberg covariant energy is equal to the expression for the Einstein energy. The discussion will involve some particular cases obtained in function of two specific parameters, and in addition presents a study of some particular solutions connected with a previous work [15].

The paper is organized as follows: in Section 2 we present the regular class of four parametric exact black hole solutions obtained by Ayón-Beato and Garcia, the Einstein, Weinberg and Møller definitions and the calculated expressions for energy. In Section 3 we perform a discussion of the results and notice some particular cases. Through the paper we consider Latin indices run from 0 to 3 , geometrized units $(G=c=1)$ and the signature $(+,-,-,-)$.

\section{ENERGY OF REGULAR EXACT BLACK HOLE SOLUTIONS COUPLED WITH NON- LINEAR ELECTRODYNAMICS SOURCE}

In a recent study [14] E. Ayón-Beato and A. Garcia developed a regular class of four parametric exact black hole solutions of Einstein's equations coupled with a nonlinear electrodynamics source. Notice that this class of solutions describes regular exact solutions under some physically reasonable assumptions. The nonlinear electrodynamics changes into the Maxwell theory in the weak 
field approximation and the corresponding solutions behave asymptotically as the Reissner-Nordström solution. This class of solution is described by four parameters, which are the mass $m$, the charge $q$, and a sort of dipole and quadrupole moments of the nonlinear source $\alpha$ and $\beta$, respectively. The $\alpha$ and $\beta$ parameters are determined by the asymptotic behaviour of the electric field. Moreover, for some particular range of the parameters the WEC energy condition is satisfied. Particular imposed values of these parameters determine a previous solution given by the authors [15].

The solution is given by

$$
d s^{2}=\left(1-\frac{2 M(r)}{r}\right) d t^{2}-\left(1-\frac{2 M(r)}{r}\right)^{-1} d r^{2}-r^{2}\left(d \theta^{2}+\sin ^{2} \theta d \varphi^{2}\right),
$$

where

$$
M(r)=\frac{m r^{\alpha}}{\left(r^{2}+q^{2}\right)^{\alpha / 2}}-\frac{q^{2} r^{\beta-1}}{2\left(r^{2}+q^{2}\right)^{\beta / 2}} .
$$

For $\alpha \geq 3, \beta \geq 4,|q| \leq 2 s_{c} m$ (with $s=|q| / 2 m$ and $s_{c}$ the critical value) these solutions describe regular charged black holes. The geometry presents a similar global structure as the Reissner-Nordström solution, but the singularity at $r=0$ has been smoothed out and $r=0$ corresponds to the origin of spherical coordinates. The regular case $|q|=2 m, \alpha \geq 1, \beta=\alpha+1$ does not correspond to a black hole and satisfies the weak energy condition only for the particular values $\alpha=1(\beta=2)$.

We compute the energy distribution of this class of solution with the Einstein, Weinberg and Møller definitions. We present the Einstein, Weinberg and Møller energy-momentum complexes [15] and the results obtained for the energy distributions. The energy component in the Einstein [1] prescription is given by

$$
E_{\text {Einstein }}=\frac{1}{16 \pi} \int \frac{\partial H_{0}{ }^{0 l}}{\partial x^{l}} d^{3} x,
$$

where $H_{0}{ }^{0 l}$ is the corresponding von Freud superpotential

$$
H_{0}{ }^{0 l}=\frac{g_{00}}{\sqrt{-g}} \frac{\partial}{\partial x^{m}}\left[(-g) g^{00} g^{l m}\right] .
$$

For performing the calculations concerning the energy component of the Einstein energy-momentum complex we have to transform the metric given by (1) in the quasi-Cartesian coordinates $(t, x, y, z)$ and obtain

$$
d s^{2}=A d t^{2}-\left(d x^{2}+d y^{2}+d z^{2}\right)-\frac{A^{-1}-1}{r^{2}}(x d x+y d y+z d z)^{2} .
$$

In spherical coordinates the nonzero components of the Einstein energy-momentum complex $H_{0}{ }^{0 l}$ are

$$
H_{0}{ }^{0 r}=\frac{2 \kappa}{r} \hat{r}-\frac{1}{A} \hat{r}(\hat{r} \cdot \nabla A)+\frac{1}{A} \nabla A
$$


where we denote $\kappa=1-A$. Using the Gauss theorem we get

$$
E_{\text {Einstein }}=\frac{1}{16 \pi} \oint H_{0}{ }^{0 r} \cdot \hat{r} r^{2} d \Omega,
$$

and the integral being taken over a sphere of radius $r$, with the outward normal $\hat{r}$ and the differential solid angle $d \Omega$. The expression for energy within radius $r$ is given by

$$
E_{\text {Einstein }}=\frac{r}{2}(1-A)=m\left(1+\frac{q^{2}}{r^{2}}\right)^{-\alpha / 2}-\frac{q^{2}}{2 r}\left(1+\frac{q^{2}}{r^{2}}\right)^{-\beta / 2} .
$$

The Weinberg energy-momentum complex [5] is defined as

$$
\tau^{\nu \lambda}=\frac{\partial}{\partial x^{\rho}} Q^{\rho \nu \lambda}
$$

where $Q^{\rho \nu \lambda}$ is the Weinberg superpotential

$$
Q^{\rho \nu \lambda}=\frac{\partial h_{\mu}^{\mu}}{\partial x_{\rho}} \eta^{\nu \lambda}-\frac{\partial h_{\mu}^{\mu}}{\partial x_{\nu}} \eta^{\rho \lambda}+\frac{\partial h^{\mu \nu}}{\partial x^{\mu}} \eta^{\rho \lambda}-\frac{\partial h^{\mu \rho}}{\partial x^{\mu}} \eta^{\nu \lambda}-\frac{\partial h^{\nu \lambda}}{\partial x_{\rho}}+\frac{\partial h^{\rho \lambda}}{\partial x_{\nu}},
$$

with $\eta_{\mu \nu}$ the Minkowski metric and $h_{\mu \nu}=g_{\mu \nu}-\eta_{\mu \nu}$. The indices on $h_{\mu \nu}$ and $\partial / \partial x^{\lambda}$ can be raised and lowered with $\eta$. For the evaluation of the energymomentum in the Weinberg prescription we use the metric transformed in quasiCartesian coordinates $(t, x, y, z)(5)$ together with the equation

$$
P^{\lambda}=\frac{1}{16 \pi} \int \frac{\partial Q^{i 0 \lambda}}{\partial x^{i}} d^{3} x
$$

The nonvanishing components $Q^{i 00}$ obtained with the Weinberg energy-momentum complex are given by

$$
Q^{i 00}=\frac{A^{\prime}}{r} \hat{r}+\frac{\hat{r}}{2}\left(\hat{r} \cdot \nabla A^{\prime}\right)-\frac{1}{2} \nabla A^{\prime},
$$

where $A^{\prime}=A^{-1}-1$. We apply the Gauss theorem and compute the energy within radius $r$

$$
E_{\text {Weinberg }}=P^{0}=\frac{1}{16 \pi} \oint Q^{i 00} n_{i} r^{2} d \Omega=\frac{r}{2} A^{\prime} .
$$

The connection between the energy component of the covariant energy-momentum four vector of the Weinberg energy-momentum complex and the energy in the Einstein prescription [15] is given by

$$
E_{\text {Weinberg }}^{\text {covariant }}=g_{00} E_{\text {Weinberg }}=\frac{\kappa r}{2}=E_{\text {Einstein }}
$$

and we have

$$
E_{\text {Weinberg }}^{\text {covariant }}=E_{\text {Einstein }}=\frac{r}{2}(1-A)=m\left(1+\frac{q^{2}}{r^{2}}\right)^{-\alpha / 2}-\frac{q^{2}}{2 r}\left(1+\frac{q^{2}}{r^{2}}\right)^{-\beta / 2} .
$$


Now, we perform the calculations in the Møller prescription. The Møller energy-momentum complex [7] is given by

$$
\Theta_{\nu}{ }^{\mu}=\frac{1}{8 \pi} \frac{\partial \chi_{\nu}{ }^{\mu \sigma}}{\partial x^{\sigma}}
$$

where the antisymmetric Møller superpotential $\chi_{\nu}{ }^{\mu \sigma}$ is defined by

$$
\chi_{\nu}^{\mu \sigma}=\sqrt{-g}\left(\frac{\partial g_{\nu \alpha}}{\partial x^{\beta}}-\frac{\partial g_{\nu \beta}}{\partial x^{\alpha}}\right) g^{\mu \beta} g^{\sigma \alpha} .
$$

The expression for energy is computed as

$$
E_{\mathrm{M} \varnothing l \mathrm{ler}}=\frac{1}{8 \pi} \int \frac{\partial \chi_{0}^{0 k}}{\partial x^{k}} d^{3} x .
$$

The Møller prescription allows to perform the calculations in any coordinate system and the required nonzero component of the Møller energy-momentum complex is

$$
\chi_{0}{ }^{0 k}=r^{2} \sin \theta \frac{d A}{d r} .
$$

The energy contained in a sphere of radius $r$ is given by

$E_{\mathrm{M} \varnothing 1 \mathrm{ler}}=\frac{r^{2}}{2} \frac{d A}{d r}=m\left(1+\frac{q^{2}}{r^{2}}\right)^{-\alpha / 2}\left(1-\frac{\alpha q^{2}}{r^{2}+q^{2}}\right)-\frac{q^{2}}{2 r}\left(1+\frac{q^{2}}{r^{2}}\right)^{-\beta / 2}\left(2-\frac{\beta q^{2}}{r^{2}+q^{2}}\right)$.

One notice the dependence of the energy in the three prescriptions on the mass $m$, the charge $q$, and the parameters $\alpha$ and $\beta$, respectively.

In the foloowing we perform a study of the conditions that have to be satisfied for obtaining the same expression for energy distribution using the Einstein, Weinberg covariant and Møller energy-momentum complexes. We impose

$$
E_{\text {Einstein }}=E_{\text {Weinberg }}^{\text {covariant }}=E_{\text {Møller }}
$$

and we obtain

$$
m\left(1+\frac{q^{2}}{r^{2}}\right)^{-\alpha / 2}\left(\frac{\alpha q^{2}}{r^{2}+q^{2}}\right)-\frac{q^{2}}{2 r}\left(1+\frac{q^{2}}{r^{2}}\right)^{-\beta / 2}\left(-1+\frac{\beta q^{2}}{r^{2}+q^{2}}\right)=0 .
$$

For the equation (22) we found the solutions $m=\frac{1}{2 r \alpha}\left(q^{2}+r^{2}\right)\left(q^{2} \frac{\beta}{q^{2}+r^{2}}-1\right) \frac{\left(\frac{q^{2}}{r^{2}}+1\right)^{\frac{1}{2} \alpha}}{\left(\frac{q^{2}}{r^{2}}+1\right)^{\frac{1}{2} \beta}}$ and numeric $\beta=24.432, r=-43.117, \alpha=-138.89, m=1.0540 \times 10^{-24}, q=$ 165. 14. We conclude that the energy calculated in Schwarzschild cartesian coordinates with the Einstein and Weinberg covariant energy-momentum complexes is equal to the energy obtained in the Møller prescription in this particular cases. 


\section{DISCUSSION}

Despite the weakness of the energy-momentum complexes, which is connected to their coordinate dependence excepting the Møller prescription, many interesting studies have been performed in the recent years. Physicists like Bondi [16], Misner [17] and Lessner [18] showed that the pseudotensorial definitions could be a reliable solution (starting point) for solving the energy-momentum localization issue.

Our work is focused on the evaluation of the energy distribution of a regular class of four parametric exact black hole solutions of Einstein's equations coupled with a nonlinear electrodynamics source given by Ayón-Beato and García. We found that the expressions for energy yielded by the Einstein, Weinberg and Møller prescriptions depend on the mass $m$, the charge $q$, and $\alpha$ and $\beta$ that represent a sort of dipole and quadrupole moments of the nonlinear source, respectively. Further, the Weinberg covariant energy distribution is the same as the Einstein energy. Also, we extend a previous work [15] and our results contain this as a particular case. In addition, we make a discussion concerning the conditions which have to be satisfied for obtaining the same expression for energy using the Einstein, Weinberg covariant and Møller energy-momentum complexes.

We present the expressions for energy obtained in these prescriptions in the following table. In addition some particular cases are discussed, and here belong the cases of the Reissner-Nordström solution (vanishing values for $\alpha$ and $\beta$ ) and Schwarzschild solution.

Our conclusions are:

a) The expressions for energy are finite in all definitions, excepting the particular case $\alpha=\beta=0, r \rightarrow 0$, which gives the same infinite result for energy distributions in the Einstein, Weinberg and Møller definitions.

b) For $\alpha=3, \beta=4$ we find the case of the regular black hole solution given by Ayón-Beato and García [19] and studied by the authors [15].

c) We observe that the particular cases $q \rightarrow 0, \alpha=\beta=0, q \rightarrow 0$ and $\alpha=\beta=0, r \rightarrow \infty$ yield the same result for energy, in all the prescriptions the energy of the black hole is equal to the ADM mass, and this result also corresponds to the Schwarzschild solution.

d) The special case $\alpha \geq 3, \beta \geq 4,|q| \leq 2 s_{c} m, r \rightarrow 0$ gives a zero value for energy in all the prescriptions, and even the expressions for energy are the same this result doesn't lead to a physically meaningful interpretation.

e) Excepting the first two cases from the table below, there is a total compatibility between the results obtained with the Einstein, Weinberg and Møller definitions. 


\begin{tabular}{|l|l|l|}
\hline Case & $E_{\text {Einstein }}=E_{\text {Weinberg }}^{\text {covariant }}$ & $E_{\text {Møller }}$ \\
\hline $\begin{array}{l}\alpha \geq 3, \beta \geq 4, \\
|q| \leq 2 s_{c} m\end{array}$ & $\begin{array}{l}m\left(1+\frac{q^{2}}{r^{2}}\right)^{-\alpha / 2}- \\
-\frac{q^{2}}{2 r}\left(1+\frac{q^{2}}{r^{2}}\right)^{-\beta / 2}\end{array}$ & $\begin{array}{l}m\left(1+\frac{q^{2}}{r^{2}}\right)^{-\alpha / 2}\left(1-\frac{\alpha q^{2}}{r^{2}+q^{2}}\right)- \\
-\frac{q^{2}}{2 r}\left(1+\frac{q^{2}}{r^{2}}\right)^{-\beta / 2}\left(2-\frac{\beta q^{2}}{r^{2}+q^{2}}\right)\end{array}$ \\
\hline$\alpha=3, \beta=4$ & $\begin{array}{l}m\left(1+\frac{q^{2}}{r^{2}}\right)^{-3 / 2}- \\
-\frac{q^{2}}{2 r}\left(1+\frac{q^{2}}{r^{2}}\right)^{-2}\end{array}$ & $\begin{array}{l}m\left(1+\frac{q^{2}}{r^{2}}\right)^{-5 / 2}\left(1-\frac{2 q^{2}}{r^{2}}\right)- \\
-\frac{q^{2}}{r}\left(1+\frac{q^{2}}{r^{2}}\right)^{-3}\left(1-\frac{q^{2}}{r^{2}}\right)\end{array}$ \\
\hline$q \rightarrow 0$ & $m$ & $m$ \\
\hline$\alpha=\beta=0,(R N)$ & $m-\frac{q^{2}}{2 r}$ & $m-\frac{q^{2}}{r}$ \\
\hline $\begin{array}{l}\alpha \geq 3, \beta \geq 4, \\
|q| \leq 2 s_{c} m, \\
r \rightarrow 0\end{array}$ & 0 & 0 \\
\hline$\alpha=\beta=0, q \rightarrow 0$ & $m$ & $m$ \\
\hline$\alpha=\beta=0, r \rightarrow 0$ & $\pm \infty$ & $\pm \infty$ \\
\hline$\alpha=\beta=0, r \rightarrow \infty$ & $m$ & $m$ \\
\hline
\end{tabular}

The equality of the results in the Einstein and Weinberg prescriptions, and in addition the equality of the results in all prescriptions in some particular cases demonstrate that the energy-momentum complexes can yield consistent expressions for energy and are useful methods for energy-momentum localization.

\section{References}

[1] A. Einstein, Preuss. Akad. Wiss. Berlin 47, 778 (1915); Addendum-ibid. 47, 799 (1915); A. Trautman, in Gravitation: an Introduction to Current Research, ed. L. Witten (Wiley, New York, 1962, p. 169).

[2] L. D. Landau and E. M. Lifshitz, The Classical Theory of Fields (Pergamon Press, 1987, p. 280).

[3] A. Papapetrou, Proc. R. Irish. Acad. A52, 11 (1948).

[4] P. G. Bergmann and R. Thompson, Phys. Rev. 89, 400 (1953).

[5] S. Weinberg, Gravitation and Cosmology: Principles and Applications of General Theory of Relativity (John Wiley and Sons, Inc., New York, 1972, p. 165).

[6] A. Qadir and M. Sharif, Phys. Lett. A167, 331 (1992).

[7] C. Møller, Ann. Phys. (NY) 4, 347 (1958).

[8] K. S. Virbhadra, Phys. Rev. D41, 1086 (1990); S. Virbhadra, Phys. Rev. D42, 2919 (1990); N. Rosen and K. S. Virbhadra, Gen. Rel. Grav. 25, 429 (1993); K. S. Virbhadra and J. C. Parikh, Phys. Lett. B331, 302 (1994); S. S. Xulu, Int. J. Theor. Phys. 37, 1773 (1998); S. S. Xulu, Int. J. Mod. 
Phys. D7, 773 (1998); E. C. Vagenas, Mod. Phys. Lett. A21, 1947 (2006); E. C. Vagenas, Mod. Phys. Lett. A19, 213 (2004); I. Radinschi, Acta Physica Slovaca, 49(5), 789 (1999); I. Radinschi, Mod. Phys. Lett. A15, Nos. 11\&12, 803 (2000); I-Ching Yang and I. Radinschi, Chin. J.Phys. 41, 326 (2003); I. Radinschi and Th. Grammenos, Int. J. Mod. Phys. A21, 2853, (2006); Th. Grammenos and I. Radinschi, Int. J. Theor. Phys. 46(4), 1055 (2007).

[9] T. Bringley, Mod. Phys. Lett. A17, 157 (2002); M. Súkeník and J. Sima, gr-qc/0101026 M. Sharif and Tasnim Fatima, Int. J. Mod. Phys. A20, 4309 (2005); M. Sharif, Nuovo Cim. B19, 463 (2004). M. Sharif, Int. J. Mod. Phys. D13, 1019 (2004); R. Gad, Mod. Phys. Lett. A19, 1847 (2004); R. Gad, Astrophys. Space Sci. 295, 451 (2005); R. Gad, Astrophys. Space Sci. 293, 453 (2004); O. Patashnick, Int. J. Mod. Phys. D14, 1607 (2005); P. Halpern, Astrophys. Space Sci. 306, 279 (2006); P. Halpern, Astrophys. Space Sci. 313 (4), 357 2008; E. C. Vagenas, Int. J. Mod. Phys. A18, 5949 (2003); E. C. Vagenas, Int. J. Mod. Phys. A18, 5781 (2003); E. C. Vagenas, JHEP 0307, 046 (2003); E. C. Vagenas, Int. J. Mod. Phys. D14, 573 (2005); Th. Grammenos, Mod. Phys. Lett., A20, 1741 (2005); T. Multamaki, A. Putaja, E. C. Vagenas and I. Vilja, Class. Quant. Grav. 25, 075017 (2008); Y.-H. Wei, Chin. Phys. Lett. 25(8), 2782 (2008); Ragab M. Gad and M. F. Mourad, Astrophys. Space Sci. 314 (4), 341 (2008); J. Matyjasek, Mod. Phys. Lett. A 23 (8), 591 (2008); G.G.L., Nashed, Chin. Phys. Lett. 25 (4), 1202 (2008); J. M. Nester, L. L. So, T. Vargas, Phys. Rev. D 78 (4), 044035 (2008).

[10] S. S. Xulu, Mod. Phys. Lett. A15, 1511 (2000); PhD Thesis, hepth/0308070; S.S. Xulu, Astrophys. Space. Sci. 23, (2003); I-Ching Yang, Ching-Tzung Yeh, Rue-Ron Hsu and Chin-Rong Lee, Int. J. Mod. Phys. D6, 349 (1997); I-Ching Yang, Wei-Fui Lin and Rue-Ron Hsu, Chin. J. Phys. 37, 113 (1999); E. C. Vagenas, Int. J. Mod. Phys. A18, 5949 (2003) ; E. C. Vagenas, Mod. Phys. Lett. A21, 1947 (2006); E. C. Vagenas, Mod. Phys. Lett. A19, 213 (2004); E. C. Vagenas, Int. J. Mod. Phys. A18, 5781 (2003); E. C. Vagenas, Int. J. Mod. Phys. D14, 573 (2005); Th. Grammenos, Mod. Phys. Lett. A20, 1741 (2005); I. Radinschi and Th. Grammenos, Int. J. Mod. Phys. A21, 2853 (2006); I. Radinschi, Mod. Phys. Lett. A15 (35), 2171 (2000); I. Radinschi, Fizika B B9(4), 203 (2000); I. Radinschi, APH N.S., Heavy Ion Physics 12, 47 (2000); I. Radinschi, Acta Physica Slovaca 50(4), 609 (2000); I. Radinschi, Chin. J. Phys. 39(3), 231 (2001); I. Radinschi, Chin. J. Phys. 39(5), 1 (2001); I. Radinschi, Mod. Phys. Lett. A16(10), 673 (2001); I-Ching Yang and I. Radinschi, Chin. J. Phys. 41, 326 (2003); M. Sharif and Tasnim Fatima, Int. J. Mod. Phys. A20, 4309 (2005); M. Sharif, Nuovo Cim. B19, 463 (2004). M. Sharif, Int. J. Mod. Phys. D13, 1019 (2004); M. Sharif and Tasnim Fatima, Nuovo Cim. B120, 533 (2005); M. Sharif and Tasnim Fatima, Astrophys. Space Sci. 302, 217 (2006); M. Sharif, M. Azam, Int. J. Mod. Phys. A22, 1935 (2007); M. Sharif, M. Jamil Amir, Can. J. Phys., 86(11), 1297 (2008); Ragab M. Gad, Mod. Phys. Lett. A19, 1847 (2004); Ragab M. Gad, Gen. 
Rel. Grav. 38, 417 (2006); Ragab M. Gad, Astrophys. Space Sci. 295, 451 (2005); Ragab M. Gad, Astrophys. Space Sci. 293, 453 (2004); Ragab M. Gad, Astrophys. Space Sci. 295, 459 (2005); Ragab M. Gad, Astrophys. Space Sci. 302141 (2006); Ragab M. Gad, Int. J. Theor.Phys. 46, 3263 (2007); O. Patashnick, Int. J. Mod. Phys. D14, 1607 (2005); Th. Grammenos and I. Radinschi, Int. J. Theor. Phys. 46, 1055 (2007); I. Radinschi and Th. Grammenos, Int. J. Theor. Phys. 47, 1363 (2008).

[11] J. M. Aguirregabiria, A. Chamorro and K. S. Virbhadra, Gen. Rel. Grav. 28, 1393 (1996); K. S. Virbhadra, Phys. Rev. D60, 104041 (1999).

[12] Gamal G. L. Nashed, Phys. Rev. D66 064015 (2002); Gamal G. L. Nashed, Nuovo Cim. 117B 521 (2002); Gamal G. L. Nashed, Int. J. Mod. Phys. Lett. A21, 3181 (2006).

[13] Chia-Chen Chang, J. M. Nester and Chiang-Mei Chen, Phys. Rev. Lett. 83, 1897 (1999).

[14] E. Ayón-Beato and A. García, Gen. Rel. Grav. 37, 635 (2005).

[15] I-Ching Yang, Chi-Long Lin and I. Radinschi, Int. J. Theor. Phys., in press

[16] H. Bondi, Proc. R. Soc. London A427, 249 (1990).

[17] C. W. Misner, K. S. Thorne and J. A. Wheeler, Gravitation, W. H. Freeman and Co., NY, 603 (1973).

[18] G. Lessner, Gen. Relativ. Gravit. 28, 527 (1996).

[19] E. Ayón-Beato and A. García, Phys. Rev. Lett. 80, 5056 (1998). 